\title{
Effect of gastrointestinal microbiome and its diversity on the expression of tumor-infiltrating lymphocytes in breast cancer
}

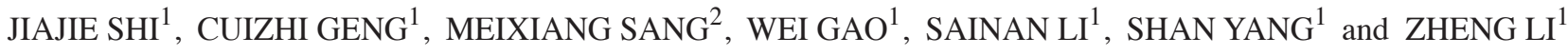 \\ Departments of ${ }^{1}$ Breast Oncology and ${ }^{2}$ Tumor Immunology, The Fourth Hospital of Hebei Medical University, \\ Shijiazhuang, Hebei 050035, P.R. China
}

Received July 17, 2018; Accepted February 22, 2019

DOI: $10.3892 / 01.2019 .10187$

\begin{abstract}
The diversity of the gastrointestinal microbiome is closely associated with human health. In the present study, the gastrointestinal microbiome and tumor-infiltrating lymphocytes (TILs) were compared in patients with breast cancer (BC). A total of 80 patients with BC were divided into three groups based on the expression of TILs, as follows: High expression of TILs (TIL-H), medium expression of TILs (TIL-M) and low expression of TILs (TIL-L). DNA of the gastrointestinal microbiome was determined by Illumina sequencing and taxonomy of $16 \mathrm{~S}$ ribosomal RNA genes. A $\chi^{2}$ test and UniFrac analysis of $\beta$-diversity were applied to assess the association between clinical characteristics and diversity of the gastrointestinal microbiome. The $\beta$-diversity distribution was statistically significant (weighted UniFrac, $\mathrm{P}<0.01$; unweighted UniFrac, $\mathrm{P}<0.01$ ) when comparing the TIL-L and TIL-H groups and when comparing the three groups (TIL-H vs. TIL-M vs. TIL-L). At the genus level, higher abundances of Mycobacterium, Rhodococcus, Catenibacterium, Bulleidia, Anaerofilum, Sneathia, Devosia and TG5, but lower abundances of Methanosphaera and Anaerobiospirillum $(\mathrm{P}<0.05)$ were identified in the TIL-L group compared with the TIL-H group. At the species level, the stercoris, barnesiae, coprophilus, flavefaciens and C21_c20 species exhibited a higher abundance in the TIL-L group, whereas producta and komagatae exhibited a greater abundance in the TIL-H group $(\mathrm{P}<0.05)$. Collectively, the diversity of the gastrointestinal microbiome was associated with the expression of TILs in patients with BC.
\end{abstract}

\section{Introduction}

Breast cancer (BC) is the most common malignant tumor and the primary cause of cancer-associated mortality in women

Correspondence to: Dr Cuizhi Geng, Department of Breast Oncology, The Fourth Hospital of Hebei Medical University, 169 Tianshan Road, Shijiazhuang, Hebei 050035, P.R. China

E-mail: cuizhigeng@hotmail.com

Key words: gastrointestinal microbiome, breast cancer, tumorinfiltrating lymphocytes, $16 \mathrm{~S}$ ribosomal DNA sequence worldwide (1). Although numerous treatments, including chemotherapy, radiotherapy, endocrine therapy and targeted therapy, are currently available for $\mathrm{BC}$, the response of patients greatly varies partly due to their own antitumor immunity (2). Accumulating evidence suggests that adaptive immunity mediated by $\mathrm{T}$ and $\mathrm{B}$ lymphocytes provides the critical foundation for effective and sustained antitumor responses. Tumor-infiltrating lymphocytes (TILs) are likely to be the most relevant indicator of tumor immunity in solid tumors, with prognostic value (3). In $\mathrm{BC}$, extensive tumor infiltration by cytotoxic $\mathrm{CD} 8^{+} \mathrm{T}$ cells is markedly associated with patient survival $(4,5)$ and response to therapy (6). Furthermore, the baseline expression of TILs can predict the pathological complete response (pCR) result following neoadjuvant chemotherapy in patients with BC (7), which is an important prognostic indicator.

Previous studies revealed that the composition of the gastrointestinal microbiome is a major environmental factor that varies among individuals, which may affect systemic immunity $(8,9)$. The gastrointestinal microbiome has been demonstrated to initiate the differentiation of $\mathrm{T}$ cells, the expansion of specific molecular subsets $(10,11)$ and the activation state of innate antigen-presenting cells (APCs), which may eventually affect priming of the systemic immune response $(12,13)$. In addition, the gastrointestinal microbiome may improve the outcomes of cancer treatment by impairing inflammatory activation in response to different therapeutic protocols (14). Numerous studies have confirmed the association between the gastrointestinal microbiome and tumors, particularly in colon carcinoma (15-17). However, for extraintestinal tumors, to the best of our knowledge, such an association has not been established. The present study aimed to assess whether the diversity of the gastrointestinal microbiome was associated with different expression patterns of TILs in patients with BC.

\section{Materials and methods}

Patients. Between March 2017 and October 2017, a total of 90 biopsy-confirmed female patients with BC were enrolled in the present study at the Breast Center of The Fourth Hospital of Hebei Medical University (Shijiazhuang, China). All patients were first treated by chemotherapy, followed by surgical treatment, as appropriate. Available clinicopathological data included age, staging, menstrual state, estrogen 
receptor (ER) and/or progesterone receptor $(\mathrm{PgR})$ status, human epidermal growth factor receptor 2 (HER2) status, TIL classification, and pCR cases. ER and PgR were assessed as positive if $\geq 1 \%$ of tumor cells exhibited nuclear staining (18). HER2-positive status was defined as a score of 3+ based on immunohistochemistry assay or HER2 gene amplification using fluorescent in situ hybridization, as described previously (19). The Miller-Payne grading system was used to evaluate the pathological response in surgical specimens (20), and $\mathrm{pCR}$ was defined as the absence of residual invasive tumor cells in the breast and axillary lymph nodes (ypT0/is + ypN0) in surgical specimens. All procedures were supervised and approved by the Human Tissue Research Committee of The Fourth Hospital of Hebei Medical University, and informed consent was provided by all participants.

Assessment of TILs using a three-grade scale. Core needle biopsy was performed in the examination room. Briefly, between three and five lump tissues were obtained from different directions to obtain a suitable number of tissue samples. Subsequently, the tissue strip was placed in $4 \%$ neutral formalin solution and sent to the Pathology Department. Evaluation of TILs on the core needle biopsy specimens was performed by two experienced pathologists who were familiar with the evaluation criteria recommended by the International TILs Working Group in 2014 (21). The whole slide was screened using a low-power field, while an area with many lymphocytes was identified as a 'hotspot'. TILs were then evaluated by light microscopy in a medium-power field (magnification, x100). The region of interest was restricted within the tumor borders as described by Salgado et al (21). TIL score was defined as the proportion of the area infiltrated by lymphocytes within the tumor itself plus the adjacent stroma, and the scores were classified as low $(<10)$, intermediate $(10-50)$ and high $(>50 \%)$, accordingly (22).

$16 S$ ribosomal DNA (rDNA) amplification. Fresh fecal samples were collected from the 90 patients with $\mathrm{BC}$ and stored at $-80^{\circ} \mathrm{C}$. DNA was extracted from all samples using a ProbeGene ${ }^{\circledR}$ Soil genomic DNA extraction kit (ProbeGene, Jiangsu, China) according to the manufacturer's protocol, and purified DNA was stored at $-80^{\circ} \mathrm{C}$ prior to further analysis.

The 16S rDNA V3-V4 region of the ribosomal RNA gene was amplified by polymerase chain reaction using primers 341F (5'-CCTACGGGNGGCWGCAG-3') and 806R (5'-GGA CTACHVGGGTATCTAAT- 3 '), where the barcode was an eight-base sequence unique to each sample. Polymerase chain reaction was performed in a 50- $\mu 1$ reaction system consisting of $5 \mu \mathrm{l}$ 10X KOD buffer, $5 \mu 12.5 \mathrm{mM}$ deoxyribonucleotide triphosphates, $1.5 \mu \mathrm{l}$ of each primer $(5 \mu \mathrm{M}), 1 \mu \mathrm{l}$ KOD polymerase (Toyobo (Shanghai) Co., Ltd., Shanghai, China) and $100 \mathrm{ng}$ template DNA. Briefly, following a denaturation step at $95^{\circ} \mathrm{C}$ for $2 \mathrm{~min}$, the amplifications were carried out with 27 cycles at a melting temperature of $98^{\circ} \mathrm{C}$ for $10 \mathrm{sec}$, an annealing temperature of $62^{\circ} \mathrm{C}$ for $30 \mathrm{sec}$, and an extension temperature of $68^{\circ} \mathrm{C}$ for $30 \mathrm{sec}$, followed by a final extension step at $68^{\circ} \mathrm{C}$ for $10 \mathrm{~min}$. Each experiment was conducted in triplicate. Amplicons were subjected to electrophoresis on $2 \%$ agarose gels, purified using the AxyPrep DNA Gel Extraction kit (Axygen; Corning Inc., Corning, NY, US), according to the manufacturer's protocol, and semi-quantified using the QuantiFluor dsDNA system (Promega Corporation, Madison, WI, US). Purified amplicons were pooled in equimolar concentrations and underwent paired-end sequencing $(2 \times 250)$ on the Illumina HiSeq2500 platform (Illumina, Inc., San Diego, CA, USA) according to the manufacturer's protocols.

Statistical analysis. Statistical analysis was performed using SPSS 23.0 software (SPSS, Inc., Chicago, IL, USA). Clinical characteristics, including age, menopausal state, staging, level of HER 2 and ER/PgR expression, were analyzed using a $\chi^{2}$ test. $\mathrm{P}<0.05$ was considered to indicate a statistically significant difference.

Raw data from Illumina sequencing contain adapters or low-quality reads that may affect subsequent data assembly and analysis. Therefore, to obtain high-quality clean reads, raw reads were filtered according to the following criteria: i) Reads containing $>10 \%$ unknown nucleotides were removed; and ii) reads containing $<80 \%$ high-quality bases (Q-value, $>20$ ) were excluded.

Paired-end clean reads were merged as raw tags using FLASH v1.2.11 (23) with a minimum overlap of $10 \mathrm{bp}$ and a mismatch error rate of $2 \%$. Noisy sequences of raw tags were filtered by QIIME v1.9.1 (24) pipeline under specific filtering conditions (25). Clean tags were searched against the reference database (http://drive5.com/uchime/ uchime_download.html) to perform reference-based chimera detection using the UCHIME algorithm (http://www.drive5. com/usearch/manual/uchime_algo.html). All chimeric tags were removed, and effective tags were finally obtained for further analysis.

The effective tags were clustered into operational taxonomic units (OTUs) of $\geq 97 \%$ similarity using the UPARSE (26) pipeline. The tag sequence with highest abundance was selected as a representative sequence within each cluster. Venn analysis was performed among groups to identify unique and common OTUs. The representative sequences were classified into organisms by a naive Bayesian model using RDP classifier v2.2 (27) based on the Greengenes (28) database (https://www. arb-silva.de/). Weighted and unweighted UniFrac distance matrices were generated using QIIME for $\beta$-diversity analysis. Between-group comparison of $\beta$-diversity was performed using Welch's t-test and Wilcoxon rank test in R. $\beta$-diversity comparison among groups was computed using Tukey's HSD test and Kruskal-Wallis H test in R. Analysis of similarity (ANOSIM) was used to test whether the differences among groups were significantly greater than those within groups. Biomarker features in each group were screened by Metastats software (v.20090414) (29).

\section{Results}

Clinical characteristics. A total of 80 patients were included in the present study (10 cases were unavailable since mass was removed in another hospital or the biopsy section could not be found) and divided into three groups as follows: High expression of TILs (TIL-H; $\mathrm{n}=21$ ), medium expression of TILs (TIL-M; $\mathrm{n}=34$ ) and low expression of TILs (TIL-L; $\mathrm{n}=25$ ). Associations between TIL distribution and clinical characteristics, including age, menstrual status, staging, HER2 
Table I. Clinical characteristics associated with TILs.

\begin{tabular}{|c|c|c|c|c|c|}
\hline \multirow[b]{2}{*}{ Characteristics } & \multicolumn{3}{|c|}{ No. of cases } & \multirow[b]{2}{*}{$\chi^{2}$} & \multirow[b]{2}{*}{ P-value } \\
\hline & TIL-low & TIL-medium & TIL-high & & \\
\hline Age (years) & & & & 0.541 & 0.736 \\
\hline$<45$ & 7 & 14 & 7 & & \\
\hline $45-59$ & 6 & 12 & 12 & & \\
\hline$\geq 60$ & 7 & 8 & 6 & & \\
\hline Staging & & & & 2.701 & 0.259 \\
\hline I & 0 & 0 & 0 & & \\
\hline II & 7 & 5 & 7 & & \\
\hline III & 11 & 20 & 12 & & \\
\hline IV & 3 & 9 & 6 & & \\
\hline Menopausal state & & & & 0.269 & 0.874 \\
\hline Yes & 11 & 19 & 15 & & \\
\hline No & 10 & 15 & 10 & & \\
\hline HER 2 & & & & 6.597 & 0.037 \\
\hline Positive & 6 & 7 & 13 & & \\
\hline Negative & 15 & 26 & 12 & & \\
\hline $\mathrm{ER} / \mathrm{PgR}$ & & & & 3.251 & 0.197 \\
\hline Positive & 18 & 25 & 15 & & \\
\hline Negative & 3 & 8 & 10 & & \\
\hline
\end{tabular}

ER, estrogen receptor; HER2, human epidermal growth factor receptor 2; PgR, progesterone receptor; TIL, tumor-infiltrating lymphocytes.

Table II. Comparison of pCR of patients in the TIL-H group and patients in the other two groups.

\begin{tabular}{lcccc}
\hline Patients & $\begin{array}{c}\text { TIL-low and } \\
\text { TIL-medium }\end{array}$ & TIL-H & $\chi^{2}$ & P-value \\
\hline pCR & 2 & 4 & 3.015 & 0.082 \\
Non-pCR & 36 & 16 & & \\
\hline
\end{tabular}

$\mathrm{H}$, high; pCR, pathological complete response; TIL, tumor-infiltrating lymphocytes.

expression and ER/PgR expression, were assessed (Table I). Only the expression status of HER2 was positively associated with TIL distribution $(\mathrm{P}=0.037)$. A total of 58 patients who underwent surgery following chemotherapy (20 from TIL-H group, 22 from TIL-M group and 16 from TIL-L group) were evaluated for chemotherapy efficiency (Table II).

Analysis of species differences. There were 3,174, 2,996 and 2,877 different OTUs in the TIL-H, TIL-M and TIL-L groups, respectively. The number of common and unique OTUs is shown in Fig. 1A, and Fig. 1B shows the top 10 species and their abundances. Tables III and IV illustrated that the gastrointestinal microbiome, when compared among the three groups (TIL-L vs. TIL-M vs. TIL-H) or compared between the TIL-L and TIL-H groups, exhibited significantly different $\beta$-diversities in weighted and unweighted UniFrac analyses, which suggested that low expression of TILs was associated with lower $\beta$-diversity $(\mathrm{P}<0.01)$. Furthermore, ANOSIM revealed a greater intergroup difference between
Table III. Weighted UniFrac distance difference analysis.

\begin{tabular}{llc}
\hline Groups & Test method & P-value \\
\hline TIL-L vs. TIL-M & t-test & $0.0004^{\mathrm{a}}$ \\
TIL-L vs. TIL-M & Wilcoxon & $0.0001^{\mathrm{a}}$ \\
TIL-L vs. TIL-H & t-test & $1.0147 \times 10^{-6 \mathrm{a}}$ \\
TIL-L vs. TIL-H & Wilcoxon & $1.8884 \times 10^{-7 \mathrm{a}}$ \\
TIL-M vs. TIL-H & t-test & 0.1227 \\
TIL-M vs. TIL-H & Wilcoxon & 0.1245 \\
TIL-L vs. TIL-M vs. TIL-H & Kruskal-Wallis & $7.2565 \times 10^{-7 a}$ \\
TIL-L vs. TIL-M vs. TIL-H & Tukey honest & $7.0541 \times 10^{-7 a}$ \\
& significant & \\
& difference & \\
\hline
\end{tabular}

${ }^{a} \mathrm{P} \leq 0.01$. H, high; L, low; M, medium; TIL, tumor-infiltrating lymphocytes.

the TIL-L and TIL-H groups compared with the intragroup difference, which indicated that the grouping was correct ( $\mathrm{P}=0.042$; Fig. 2).

Relative abundance of microbiota in TIL-H and TIL-L groups. The different distributions of microbiota in TIL-H and TIL-L groups were assessed using Metastats software. Table V demonstrated that At the genus level, patients in the TIL-L group had higher abundances of Mycobacterium, Rhodococcus, Catenibacterium, Bulleidia, Anaerofilum, Sneathia, Devosia and TG5, but lower abundances of Methanosphaera and Anaerobiospirillum compared with the TIL-H group $(\mathrm{P}<0.05)$. At the species level, the abundances of stercoris, barnesiae, coprophilus, flavefaciens and C21_c20 were greater in the 

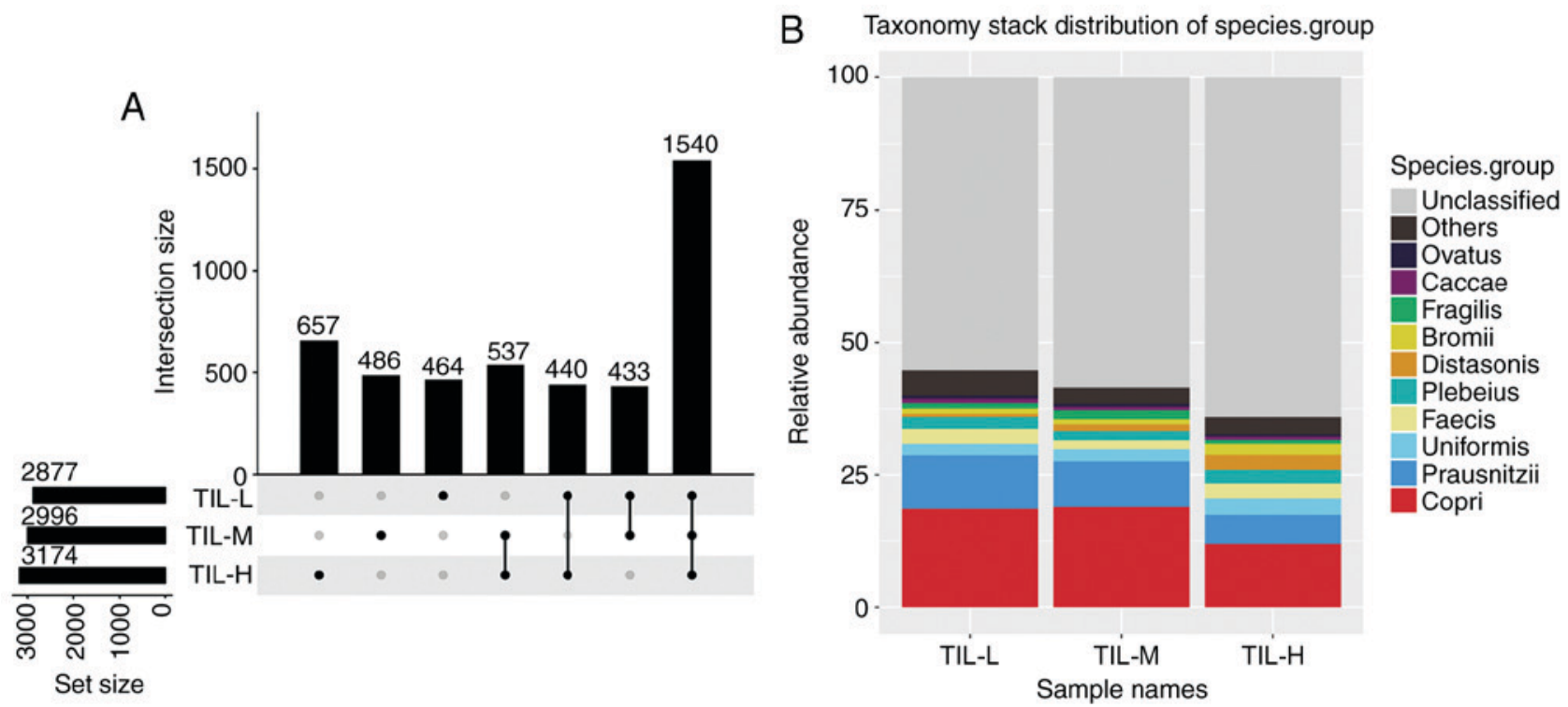

Figure 1. Operational taxonomic unit quantitative differences and species distribution stacking maps for the three groups. (A) Each point represents a group, and the lines between points represent the intersection of two points. (B) Species composition of each sample at the species level was assessed; the species abundance of different samples is presented. Only the top 10 species are shown, while the remaining species were classified into the 'others' category. Tags that could not be annotated at that level were classified into the 'unclassified' category. H, high; L, low; M, medium; TIL, tumor-infiltrating lymphocytes.

Table IV. Unweighted UniFrac distance difference analysis.

\begin{tabular}{llc}
\hline Groups & Test method & P-value \\
\hline TIL-L vs. TIL-M & t-test & 0.4007 \\
TIL-L vs. TIL-M & Wilcoxon & 0.1997 \\
TIL-L vs. TIL-H & t-test & $1.2397 \times 10^{-12 a}$ \\
TIL-L vs. TIL-H & Wilcoxon & $7.5180 \times 10^{-12 a}$ \\
TIL-M vs. TIL-H & t-test & $4.9195 \times 10^{-12 a}$ \\
TIL-M vs. TIL-H & Wilcoxon & $2.9383 \times 10^{-12 a}$ \\
TIL-L vs. TIL-M vs. TIL-H & Kruskal-Wallis & $7.0570 \times 10^{-14 a}$ \\
TIL-L vs. TIL-M vs. TIL-H & Tukey honest & $1.9653 \times 10^{-15 a}$ \\
& significant & \\
& difference & \\
\hline
\end{tabular}

${ }^{\mathrm{a}} \mathrm{P} \leq 0.01 . \mathrm{H}$, high; L, low; M, medium; TIL, tumor-infiltrating lymphocytes.

TIL-L group, while the abundances of producta and komagatae were greater in the TIL-H group $(\mathrm{P}<0.05)$.

\section{Discussion}

Recently, increasing attention has been paid to the effects of the gastrointestinal microbiome on human diseases. Diversity of the gastrointestinal microbiome is closely associated with human health, including immunity, digestion, obesity (30), diabetes $(31,32)$, heart disease $(33,34)$, acquired immune deficiency syndrome (35) and cancer $(36,37)$.

The present study demonstrated that the gastrointestinal microbiome was distinctly diverse and compositionally different among different TIL expression groups of patients with BC. Higher TIL expression was associated with a greater diversity of the gastrointestinal microbiome in the present study. A previous study only suggested that patients with $\mathrm{BC}$ possess statistically different microbiota composition compared with

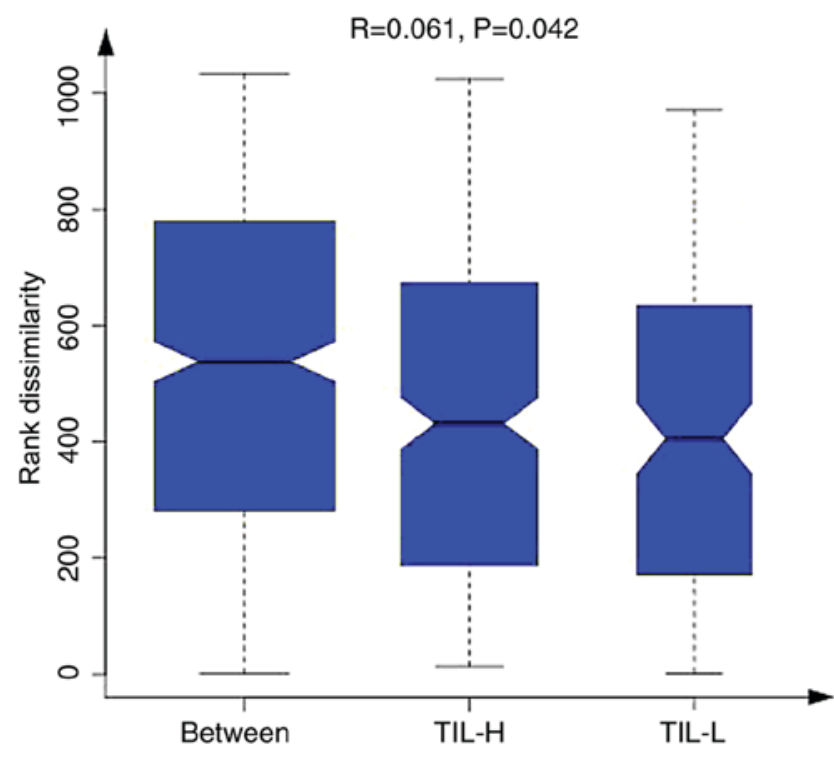

Figure 2. When comparing intragroup and intergroup Ranks mean values, information of grouping differences can be obtained using a box diagram method. When the dissimilarity rank between the groups was higher than that within the groups, the intergroup difference was greater than the intragroup difference. H, high; L, low; TIL, tumor-infiltrating lymphocytes.

controls (38), and the gastrointestinal microbiome is associated with the clinical or biological characteristics of patients with $\mathrm{BC}(38,39)$. However, the present study revealed that microbiome diversity was associated with TIL distribution. Additionally, differentially expressed microbiota species among different TIL groups were identified. Among the gastrointestinal microbiome, barnesiae and coprophilus belong to the genus Bacteroides that can modulate estrogen metabolism and function as a risk factor for BC (40-43); in this study, higher abundance of barnesiae was associated with the low expression of TILs., indicating barnesiae could be a risk factor for 


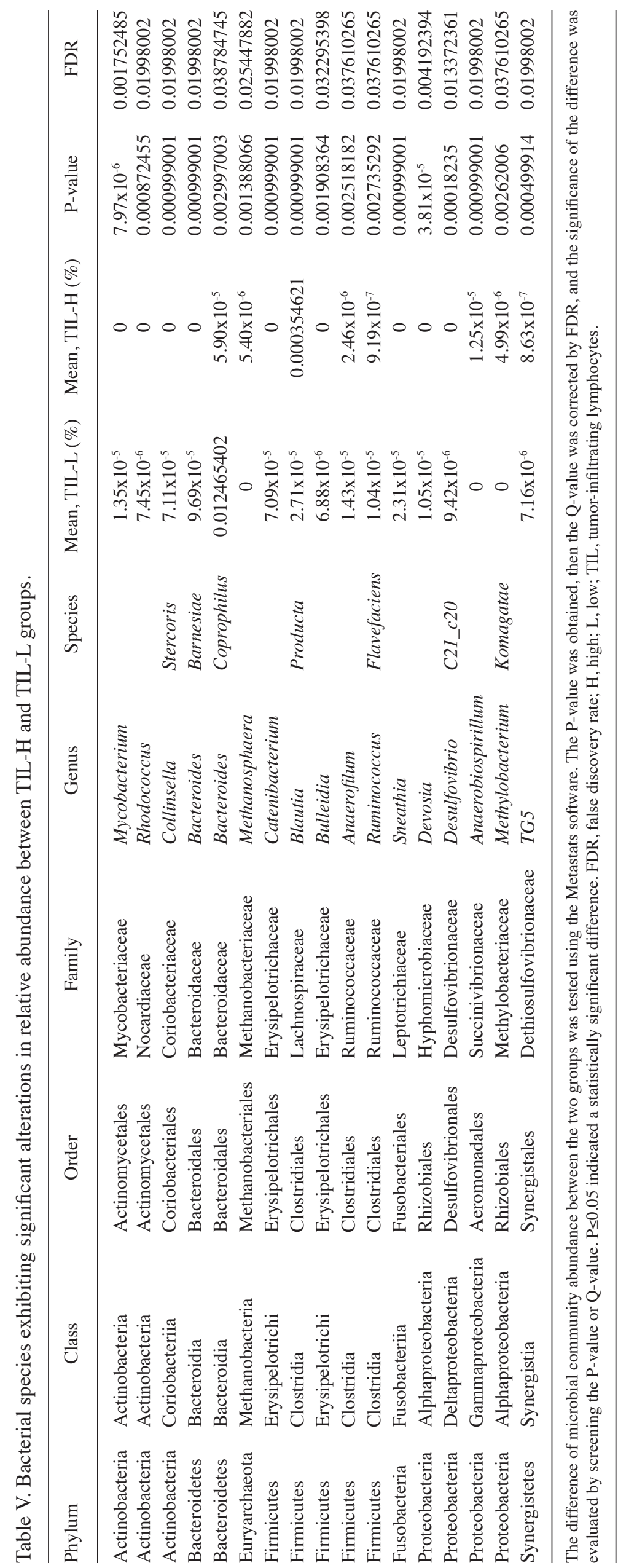


$\mathrm{BC}$. The mechanism underlying barnesiae-modulated estrogen metabolism in response to immunity modification in $\mathrm{BC}$ remains unclear. The state of TIL expression in situ exhibits a strong association with the outcomes and treatment efficiency of patients with $\mathrm{BC}$, and the gastrointestinal microbiome can regulate immune activation, following treatment with chemotherapeutic agents $(14,44-46)$. The results of the present study revealed that greater quantity and abundance of the gastrointestinal microbiome were positively associated with the expression of TILs, demonstrating an internal link between the microbiome and immunity in the pathogenesis of BC. Therefore, the treatment efficiency should be assessed in a cohort consisting of large-scale samples.

Patients with triple-negative BC and HER2-positive BC may benefit from neoadjuvant chemotherapy (47), and the data of the present study revealed that HER2 expression was positively associated with high TIL expression. Furthermore, patients with high TIL expression exhibited good outcomes following chemotherapy. All these findings implied an inherent link among microbiome, immunity and treatment efficiency in patients with $\mathrm{BC}$.

The small sample size is the main limitation of the present study. Additionally, further studies regarding the mechanism need to be performed in the future. In these, a $\mathrm{BC}$ mouse model will be established to verify the conclusions of the present study by altering the gastrointestinal microbiome.

In conclusion, expression levels of TILs were associated with the diversity of the gastrointestinal microbiome in patients with BC. The results of the present study suggested that the gastrointestinal microbiome may affect the prognosis of patients with $\mathrm{BC}$ by interacting with TIL expression.

\section{Acknowledgements}

The authors would like to thank Professor Zhanjun Guo (Rheumatic Immunology Department), Dr Xi Zhang and Dr Meng Cheng (Breast Oncology Department) from The Fourth Hospital of Hebei Medical University (Shijiazhuang, China) for their help in reviewing this manuscript.

\section{Funding}

The present study was supported by grants from Hebei Science and Technology Planning (grant no. 16967788D).

\section{Availability of data and materials}

The datasets used and/or analyzed during the present study are available from the corresponding author on reasonable request.

\section{Authors' contributions}

CG designed the experiment, provided financial support, revised the manuscript and gave final approval of the version to be published. MS was responsible for the interpretation of data. JS and WG performed the experiments, acquired the data and wrote the paper. SL, SY and ZL made substantive contributions to the work, including data collecting and manuscript revising.

\section{Ethics approval and consent to participate}

Ethical approval for this project was obtained from the Ethics Committee of The Fourth Hospital of Hebei Medical University. Informed consent was provided by all participants. All procedures involving human participants were performed in accordance with the ethical standards of the institutional and/or national research committee and with the 1964 Helsinki Declaration and its later amendments or comparable ethical standards.

\section{Patient consent for publication}

The patients' data were anonymized, and hospital numbers and associated data may be provided only for scientific purposes. All patients provided written informed consent for their data to be published.

\section{Competing interests}

The authors declare that they have no competing interests.

\section{References}

1. Akram M, Iqbal M, Daniyal M and Khan AU: Awareness and current knowledge of breast cancer. Biol Res 50: 33, 2017.

2. de la Cruz-Merino L, Chiesa M, Caballero R, Rojo F, Palazón N, Carrasco FH and Sánchez-Margalet V: Breast cancer immunology and immunotherapy: Current status and future perspectives. Int Rev Cell Mol Biol 331: 1, 2017.

3. Gooden MJ, de Bock GH, Leffers N, Daemen T and Nijman HW: The prognostic influence of tumour-infiltrating lymphocytes in cancer: A systematic review with meta-analysis. Br J Cancer 105: 93-103, 2011.

4. Mahmoud SM, Paish EC, Powe DG, Macmillan RD, Grainge MJ, Lee AH, Ellis IO and Green AR: Tumor-infiltrating CD8+ lymphocytes predict clinical outcome in breast cancer. J Clin Oncol 29: 1949-1955, 2011.

5. Liu S, Foulkes WD, Leung S, Gao D, Lau S, Kos Z and Nielsen TO: Prognostic significance of FOXP3+ tumor infiltrating lymphocytes in breast cancer depends on estrogen receptor and human epidermal growth factor receptor-2 expression status and concurrent cytotoxic T-cell infiltration. Breast Cancer Res 16: 432, 2014.

6. Seo AN, Lee HJ, Kim EJ, Kim HJ, Jang MH, Lee HE, Kim YJ, Kim JH and Park SY: Tumour-infiltrating CD8+ lymphocytes as an independent predictive factor for pathological complete response to primary systemic therapy in breast cancer. Br J Cancer 109: 2705-2713, 2013.

7. Denkert C, Loibl S, Noske A, Roller M, Muller BM, Komor M, Budczies J, Darb-Esfahani S, Kronenwett R, Hanusch C, et al: Tumor-associated lymphocytes as an independent predictor of response to neoadjuvant chemotherapy in breast cancer. J Clin Oncol 28: 105-113, 2010.

8. Spranger S, Sivan A, Corrales L and Gajewski TF: Tumor and host factors controlling antitumor immunity and efficacy of cancer immunotherapy. Adv Immunol 130: 75-93, 2016.

9. Qin Q, Miao J, Wang S, Yu Q, Li M, He F and Wang G: Association between intestinal flora and immunity in middle-aged and aged people by PCR-DGGE. Wei Sheng Yan Jiu 46: 40-45, 2017 (In Chinese).

10. Hooper LV, Littman DR and Macpherson AJ: Interactions between the microbiota and the immune system. Science 336: 1268-1273, 2012.

11. Ivanov II and Honda K: Intestinal commensal microbes as immune modulators. Cell Host Microbe 12: 496-508, 2012.

12. Abt MC, Osborne LC, Monticelli LA, Doering TA, Alenghat T, Sonnenberg GF, Paley MA, Antenus M, Williams KL, Erikson J, et al: Commensal bacteria calibrate the activation threshold of innate antiviral immunity. Immunity 37: 158-170, 2012.

13. Ganal SC, Sanos SL, Kallfass C, Oberle K, Johner C, Kirschning C, Lienenklaus S, Weiss S, Staeheli P, Aichele P and Diefenbach A: Priming of natural killer cells by nonmucosal mononuclear phagocytes requires instructive signals from commensal microbiota. Immunity 37: 171-186, 2012. 
14. Iida N, Dzutsev A, Stewart CA, Smith L, Bouladoux N, Weingarten RA, Molina DA, Salcedo R, Back T, Cramer S, et al: Commensal bacteria control cancer response to therapy by modulating the tumor microenvironment. Science 342: 967-970, 2013.

15. Dingemanse $C$, Belzer $C$, van Hijum SA, Günthel $M$, Salvatori D, den Dunnen JT, Kuijper EJ, Devilee P, de Vos WM, van Ommen GB and Robanus-Maandag EC: Akkermansia muciniphila and Helicobacter typhlonius modulate intestinal tumor development in mice. Carcinogenesis 36: 1388-1396, 2015

16. Omar Al-Hassi H, Ng O and Brookes M: Tumour-associated and non-tumour-associated microbiota in colorectal cancer. Gut 67 : 395, 2018.

17. Peled JU, Devlin SM, Staffas A, Lumish M, Khanin R, Littmann ER, Ling L, Kosuri S, Maloy M, Slingerland JB, et al: Intestinal microbiota and relapse after hematopoietic-cell transplantation. J Clin Oncol 35: 1650-1659, 2017.

18. Hammond ME, Hayes DF, Dowsett M, Allred DC, Hagerty KL, Badve S, Fitzgibbons PL, Francis G, Goldstein NS, Hayes M, et al: American society of clinical oncology/college of American pathologists guideline recommendations for immunohistochemical testing of estrogen and progesterone receptors in breast cancer. J Clin Oncol 28: 2784-2795, 2010.

19. Wolff AC, Hammond MEH, Allison KH, Harvey BE, Mangu PB, Bartlett JMS, Bilous M, Ellis IO, Fitzgibbons P, Hanna W, et al: Human epidermal growth factor receptor 2 testing in breast cancer: American society of clinical oncology/college of American pathologists clinical practice guideline focused update. J Clin Oncol 36: 2105-2122, 2018.

20. Ogston KN, Miller ID, Payne S, Hutcheon AW, Sarkar TK, Smith I, Schofield A and Heys SD: A new histological grading system to assess response of breast cancers to primary chemotherapy: prognostic significance and survival. Breast 12: 320-327, 2003.

21. Salgado R, Denkert C, Demaria S, Sirtaine N, Klauschen F, Pruneri G, Wienert S, Van den Eynden G, Baehner FL, Penault-Llorca F, et al: The evaluation of tumor-infiltrating lymphocytes (TILs) in breast cancer: recommendations by an International TILs Working Group 2014. Ann Oncol 26: 259-271, 2015.

22. Hida AI and Ohi Y: Evaluation of tumor-infiltrating lymphocytes in breast cancer; proposal of a simpler method. Ann Oncol 26: 2351,2015

23. Magoč T and Salzberg SL: FLASH: Fast length adjustment of short reads to improve genome assemblies. Bioinformatics 27: 2957-2963, 2011

24. Caporaso JG, Kuczynski J, Stombaugh J, Bittinger K, Bushman FD, Costello EK, Fierer N, Peña AG, Goodrich JK, Gordon JI, et al: QIIME allows analysis of high-throughput community sequencing data. Nat Methods 7: 335-336, 2010.

25. Bokulich NA, Subramanian S, Faith JJ, Gevers D, Gordon JI, Knight R, Mills DA and Caporaso JG: Quality-filtering vastly improves diversity estimates from Illumina amplicon sequencing. Nat Methods 10: 57-59, 2013.

26. Edgar and Robert C: UPARSE: highly accurate OTU sequences from microbial amplicon reads. Nat Methods 10: 996-998, 2013.

27. Wang Q, Garrity GM, Tiedje JM and Cole JR: Naive Bayesian classifier for rapid assignment of rRNA sequences into the new bacterial taxonomy. Appl Environ Microbiol 73: 5261-5267, 2007.

28. DeSantis TZ, Hugenholtz P, Larsen N, Rojas M, Brodie EL, Keller K, Huber T, Dalevi D, Hu P and Andersen GL: Greengenes, a chimera-checked 16S rRNA gene database and workbench compatible with ARB. Appl Environ Microbiol 72: 5069-5072, 2006.

29. White JR, Nagarajan N and Pop M: Statistical methods for detecting differentially abundant features in clinical metagenomic samples. PLoS Comput Biol 5: e1000352, 2009.

30. Cotillard A,Kennedy SP, Kong LC,PriftiE, Pons N, Le ChatelierE, Almeida M, Quinquis B, Levenez F, Galleron N, et al: Dietary intervention impact on gut microbial gene richness. Nature 500 $585-588,2013$

31. Larsen N, Vogensen FK, van den Berg FW, Nielsen DS, Andreasen AS, Pedersen BK, Al-Soud WA, Sørensen SJ, Hansen LH and Jakobsen M: Gut microbiota in human adults with type 2 differs from non-diabetic adults. PLoS One 5: e90855, 2000.
32. Markle JG, Frank DN, Mortin-Toth S, Robertson CE, Feazel LM, Rolle-Kampczyk U, von Bergen M, McCoy KD, Macpherson AJ and Danska JS: Sex differences in the gut microbiome drive hormone-dependent regulation of autoim munity. Science 339: 1084-1088, 2013.

33. Koeth RA, Wang Z, Levison BS, Buffa JA, Org E, Sheehy BT, Britt EB, Fu X, Wu Y, Li L, et al: Intestinal microbiota metabolism of $\mathrm{L}$ carnitine, a nutrient in red meat promotes atherosclerosis. Nat Med 19: 576-585, 2013

34. Wang T, Cai G, Qiu Y, Fei N, Zhang M, Pang X, Jia W, Cai S and Zhao L: Structural segregation of gut microbiota between colorectal cancer patients and healthy volunteers. ISME J 6: 320-329, 2011.

35. Vujkovic-Cvijin I, Dunham RM, Iwai S, Maher MC, Albright RG, Broadhurst MJ, Hernandez RD, Lederman MM, Huang Y, Somsouk M, et al: Dysbiosis of the gut microbiota is associated with HIV disease progression and tryptophan catabolism. Sci Transl Med 5: 193ra91, 2013.

36. Fox JG, Feng Y, Theve EJ, Raczynski AR, Fiala JLA, Doernte AL, Williams M, McFaline JL, Essigmann JM, Schauer DB, et al: Gut microbes define liver cancer risk in mice exposed to chemical and viral trans genic hepatocarcinogens. Gut 59: 88-97, 2009

37. Wang Z, Klipfell E, Bennett BJ, Koeth R, Levison BS, Dugar B, Feldstein AE, Britt EB, Fu X, Chung YM, et al: Gut flora metabolism of phosphatidylcholine promotes cardiovascular disease. Nature 472: 57-63, 2011

38. Goedert JJ, Jones G, Hua X, Xu X, Yu G, Flores R, Falk RT, Gail MH, Shi J, Ravel J and Feigelson HS: Investigation of the association between the fecal microbiota and breast cancer in postmenopausal women: a population-based case-control pilot study. J Natl Cancer Inst 107: djv147, 2015.

39. Luu TH, Michel C, Bard JM, Dravet F, Nazih H and Bobin-Dubigeon C: Intestinal proportion of Blautia sp. is associated with clinical stage and histoprognostic grade in patients with early-stage breast cancer. Nutr Cancer 69: 267-275, 2017.

40. Fuhrman BJ, Feigelson HS, Flores R, Gail MH, Xu X, Ravel J and Goedert JJ: Associations of the fecal microbiome with urinary estrogens and estrogen metabolites in postmenopausal women. J Clin Endocrinol Metab 99: 4632-4640, 2014.

41. Flores R, Shi J, Fuhrman B, Xu X, Veenstra TD, Gail MH, Gajer P, Ravel J and Goedert JJ: Fecal microbial determinants of fecal and systemic estrogens and estrogen metabolites: A cross-sectional study. J Transl Med 10: 253, 2012.

42. Estrogen excretion patterns and plasma levels in vegetarian and omnivorous women. Nutr Rev 41: 180-183, 1983.

43. Goldin BR, Adlercreutz H, Gorbach SL, Warram JH, Dwyer JT, Swenson L and Woods MN: Estrogen excretion patterns and plasma levels in vegetarian and omnivorous women. N Engl J Med 307: 1542-1547, 1982.

44. Savas P, Salgado R, Denkert C, Sotiriou C, Darcy PK, Smyth MJ and Loi S: Clinical relevance of host immunity in breast cancer: from TILs to the clinic. Nat Rev Clin Oncol 13: 228-241, 2016.

45. Viaud S, Saccheri F, Mignot G, Yamazaki T, Daillère R, Hannani D, Enot DP, Pfirschke C, Engblom C, Pittet MJ, et al: The intestinal microbiota modulates the anticancer immune effects of cyclophosphamide. Science 342: 971-976, 2013.

46. Galon J, Costes A, Sanchez-Cabo F, Kirilovsky A, Mlecnik B, Lagorce-Pagès C, Tosolini M, Camus M, Berger A, Wind P, et al: Type, density, and location of immune cells within human colorectal tumors predict clinical outcome. Science 313: 1960-1964, 2006.

47. Rastogi P, Anderson SJ, Bear HD, Geyer CE, Kahlenberg MS, Robidoux A, Margolese RG, Hoehn JL, Vogel VG, Dakhil SR, et al: Preoperative chemotherapy: Updates of national surgical adjuvant breast and bowel project protocols B-18 and B-27. J Clin Oncol 26: 778-785, 2008.

This work is licensed under a Creative Commons Attribution-NonCommercial-NoDerivatives 4.0 International (CC BY-NC-ND 4.0) License. 